\title{
Preparation, High-Density Spherical, and Low Sensitivity of RDX/NC/PMMA Composite Particles
}

\author{
Xinlei Jia $\mathbb{D}^{1,},{ }^{1,2}$ Wenshan Peng $\left(\mathbb{D},{ }^{2}\right.$ Lanjuan Xu, ${ }^{1}$ Qing Cao, ${ }^{1}$ Shaohua Xing, ${ }^{2}$ Guangyi Liu, ${ }^{2}$ \\ Xiaoheng Geng, ${ }^{1,2}$ Haiying Guo, ${ }^{1}$ Guojun Han, ${ }^{1}$ and Jingyu Wang ${ }^{3}$ \\ ${ }^{1}$ College of Chemical Engineering and Safety, Binzhou Key Laboratory of Chemical Process Safety, Binzhou University, Binzhou, \\ Shandong 256600, China \\ ${ }^{2}$ State Key Laboratory for Marine Corrosion and Protection, Luoyang Ship Material Research Institute (LSMRI), Wenhai Road, \\ Qingdao 266237, China \\ ${ }^{3}$ School of Environment and Safety Engineering, Shanxi Engineering Technology Research Center for Ultrafine Powder, \\ North University of China, Taiyuan, Shanxi 030051, China
}

Correspondence should be addressed to Xinlei Jia; 1004024260@qq.com and Wenshan Peng; pengwenshan1386@126.com

Received 2 June 2021; Revised 9 July 2021; Accepted 8 September 2021; Published 18 October 2021

Academic Editor: Bhanu P. Singh

Copyright (c) 2021 Xinlei Jia et al. This is an open access article distributed under the Creative Commons Attribution License, which permits unrestricted use, distribution, and reproduction in any medium, provided the original work is properly cited.

In order to reduce the mechanical sensitivity of cyclotrimethylenetrinitramine (RDX) and improve its energy density, spherical RDX/PMMA/NC composite particles were prepared by spray drying method, selecting polymethyl methacrylate (PMMA, hydrocarbon binder) with excellent mechanical properties and nitrocellulose (NC, energy-containing binder) with higher density as the desensitizing shell material, $\mathrm{RDX}$ as the core material. PMMA/NC composite particles of the same component were prepared by a simple solvent evaporation method at the same time. Structural characterizations and thermal stability of the composites were systematically studied by scanning electron microscopy (SEM), X-ray diffraction (XRD), and differential scanning calorimeter (DSC), respectively. Moreover, the safety performance has been qualitatively tested and analyzed through impact sensitivity and friction sensitivity. The results show that the addition of NC/PMMA binder would not change the original crystal structure of RDX, and the RDX/NC/PMMA composites fabricated by spray drying presented spherical particles with uniform distribution and smooth surface. The $\mathrm{T}_{\mathrm{p} 0}$ of RDX/NC/PMMA composite particles increased from $220.3^{\circ} \mathrm{C}$ to $228.2^{\circ} \mathrm{C}$, improving the thermal decomposition performance. The $\mathrm{H}_{50}$ rose from $29.32 \mathrm{~cm}$ to $84.3 \mathrm{~cm}$, and the probability of friction explosion decreased from $96 \%$ to $8 \%$, significantly enhancing the safety performance. In short, the RDX/NC/PMMA composites prepared via the spray drying method and the improvement of their performance have positive significance for the development of explosives in pursuit of high energy and low sensitivity.

\section{Introduction}

In the past decade, the diversification and refinement of the energy output structure of explosives, the composite, nanometer, and spheroidization of explosive reaction materials, and the sensitization of explosive charges have become the research focus [1-3]. The excellent properties of RDX such as explosion heat, explosion volume, and explosion velocity were widely utilized in modified double-click propellants and propellants. However, the defects of industrial RDX such as rough particle surface, many internal defects, and poor safety, which had become the bottleneck of wider application [4]. The composite energetic particles are prepared by technical means such as mechanical ball milling compound [5], sol-gel method [6], vapor precipitation method [7], and other technical methods to increase energy density and reduce sensitivity. Due to the synergistic effect and the simultaneous toughening enhancement effect [8], 
the comprehensive performance of the prepared composite energetic particles has been improved to a certain extent. Extraordinarily the composite modification of organic polymer by explosive particles, the shock properties of the polymer can be integrated by the modified composite particles, and enhance the reinforcing and toughening when the explosive particles were exerted in the meantime.

At present, foreign research on inductive reduction mechanisms and methods mainly focused on how to reduce the generation of hotspots, reduce the temperature of hotspots, and prevent hotspots from spreading after they are generated and causing explosions $[9,10]$. The spheroidization of explosive particles can retard the formation of hot spots after the explosive was subjected to external energy, which was beneficial to reduce the mechanical sensitivity of the explosive and improve the charge performance at the same time. In other words, since particles with irregular shapes are characterized by uneven surfaces, which have a host of edges and corners and have serious mutual friction between particles, stress concentration is prone to occur and hot spots are formed in rough areas. However, the spherical composite explosive particles are characterized by a relatively smooth surface and a dense interior. The friction between and inside the particles is tiny, and hot spots are no picnic to form. It is precisely because of advantages in large numbers of spherical explosives that a growing number of researchers are keen to study the sphericalization of composite explosives [11-13]. Among them, the spray-drying method can be used to modify the surface of explosives, which is an effective means for large-scale preparation of core-shell nanocomposite particles and has brilliant application prospects. Qiu et al. prepared spherical RDX particles by spray drying, which had lower shock wave sensitivity [14], it was reported that spray drying technology may be suitable for the preparation of a wide range of insensitive explosive compositions at the same time, and this was correctly verified in the later stage certainly. Ji et al. prepared HMX/F2602 spherical nanoscale composite material by spray drying method, whose thermal stability was significantly improved, and its impact sensitivity was obviously reduced because its $\mathrm{H}_{50}$ (features fall- high) value was increased from $18.20 \mathrm{~cm}$ to $35.37 \mathrm{~cm} \mathrm{[15].} \mathrm{Bian} \mathrm{et} \mathrm{al.}$ reported a new method for recrystallization of CL-20 from irregular bulk energetic materials EMs using a surfactantassisted self-assembly process to produce uniform spherical micron-sized particles and prepared submicron spherical CL-20 particles with good comprehensive performance and stable crystal form [16]. Li et al. prepared submicron iron tannate/nitramine explosive composite microspheres with spherical particles and tremendous dispersibility by spray drying, with a particle size of $500 \sim 1000 \mathrm{~nm}$, and the activation energy of Ta-Fe/CL-20 composite microspheres was reduced by $9.6 \mathrm{~kJ} \bullet \mathrm{mol}-1$ compared with the raw material CL-20, and its thermal decomposition catalytic effect was obvious. Since the hydrocarbon binder does not contain energy, it has a certain insensitive effect and has good thermal stability and blood return stability, so the composite energetic microspheres with good morphology and low sensitivity can be prepared by spray drying technology; how- ever, the density of the binder is little, and the energy density of the system will be reduced by excessive addition. Since the hydrocarbon binder does not contain energy and has considerable passivation, good thermal stability, and blood return stability, it is possible to prepare composite energetic microspheres with good morphology and low sensitivity by spray drying technology. It is worth noting that the density of the binder is little, and the energy density of the system will be reduced by excessive addition. As researchers explored the desensitization of energetic materials, they found that polymethyl methacrylate (PMMA) is a thermoplastic polymer with excellent performance and has good comprehensive mechanical properties, and nitrocellulose (NC) is characterized by good mechanical strength, good toughness, fast drying speed, and good compatibility with various plasticizers and adhesives. Notably, it is necessary to maintain a certain energy output by adding energetic binders; however, the thermal stability, chemical stability, and compatibility of energetic binders were lower than other binders, so the combination of hydrocarbon binders and energetic binders was of great significance to the desensitization of energetic materials.

In our paper, we selected polymethyl methacrylate with excellent mechanical properties and nitrocellulose with higher density as the desensitizing shell materials, RDX as the core material. RDX/PMMA/NC composite energetic microspheres with uniform particle size distribution, excellent thermal performance, and low sensitivity were prepared by spray drying technology. And the apparent activation energy during the thermal decomposition reaction of the energetic material was obtained through the DSC curves to verify the thermal stability of the composite energetic microspheres. This report has positive significance for the development of composite energetic materials with high energy density and low sensitivity.

\section{Experiment Parts}

2.1. Reagents and Instruments. Field-emission scanning electron microscopy (FESEM) images were taken on a MIRA3 LMH SEM (Tescan) at a magnification of $10 \mathrm{k}$. X-ray diffraction (XRD) patterns were obtained using a DX-2700 (Dandong Haoyuan Corporation, Liaoning, China) X-ray diffractometer with $\mathrm{Cu}-\mathrm{K} \alpha(40 \mathrm{kV}, 30 \mathrm{~mA})$ radiation at $\lambda=$ $1.5418 \AA$. All samples were scanned from $5^{\circ}$ to $50^{\circ}$ with steps 0.03 and $6 \mathrm{~s}$ counting time. Thermal analysis was performed on a differential scanning calorimeter (DSC-131, France Setaram Corporation, Shanghai, China) at heating rates of 5,10 , and $20^{\circ} \mathrm{C} / \mathrm{min}$. The impact sensitivity was tested with a homebuilt type 12 drop hammer apparatus. The special height $\left(\mathrm{H}_{50}\right)$ represents the height from which $2.500 \pm$ $0.002 \mathrm{~kg}$ drop-hammer will result in an explosive event in $50 \%$ of the trials. In each determination, 25 drop tests were made to calculate the $\mathrm{H}_{50}$.

2.2. Preparation of $R D X / N C / P M M A$. The principle of preparing RDX/NC/PMMA composite particles by spray drying technology was that the RDX/NC/PMMA cosolution was impacted by a high-speed flowing inert gas (Nitrogen), 


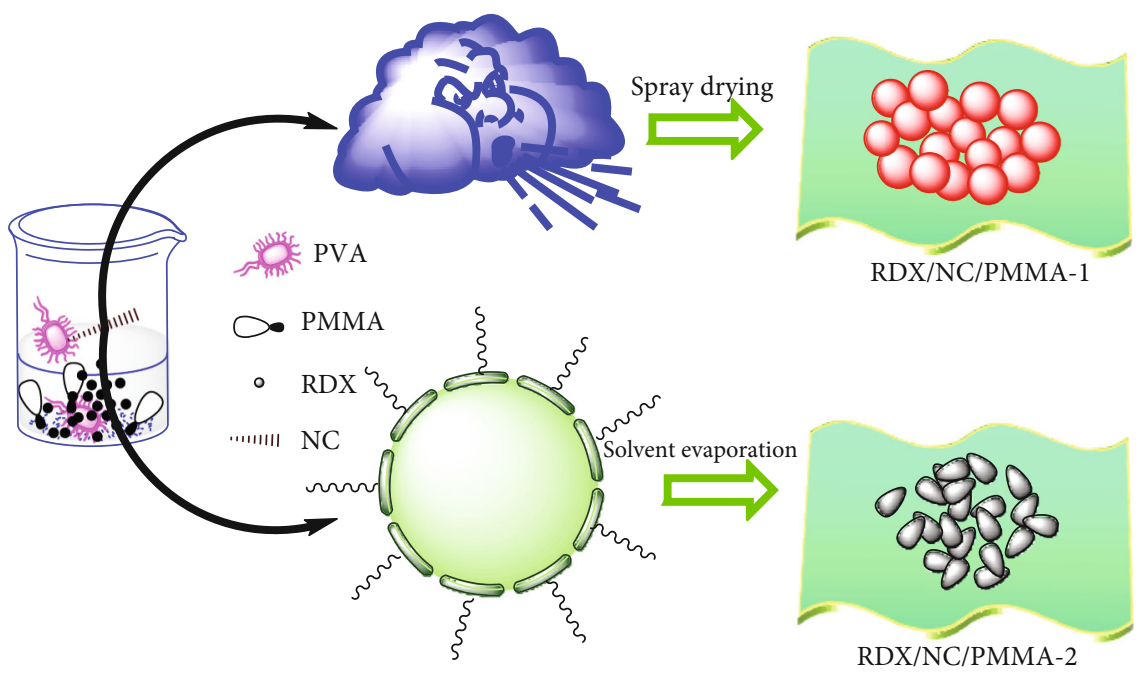

FIGURE 1: Schematic diagram of the preparation process of RDX/NC/PMMA.

so that a relatively large friction force was generated on the liquid surface of the liquid droplet, the liquid droplet was dispersed into a plurality of small liquid droplets, and the small liquid droplets and nitrogen gas form a liquid film. Subsequently, the droplets expanded and ruptured, and the particles were shaped when the solvent evaporates. The operation flow is shown in Figure 1.

(1) Preparation of precursor mixed solution. Dissolve $5 \mathrm{~g}$ RDX and $0.2 \mathrm{~g} \mathrm{NC}$ in $40 \mathrm{ml}$ acetone solution, stir magnetically at $65^{\circ} \mathrm{C}$ for $5 \mathrm{~h}$, and wait until RDX and $\mathrm{NC}$ are completely dissolved to form a uniform RDX/NC precursor solution; (2) add $0.1 \mathrm{~g}$ PMMA to the precursor solution prepared of the first step and continue stirring until it was completely dissolved to form a stabilized and transparent RDX/NC/PMMA solution; (3) a drop of $3 \%$ polyvinyl alcohol (PVA) dispersant was dropped into the solution, and the solution was shaken by ultrasound at a frequency of $40 \mathrm{~Hz}$ for $30 \mathrm{~min}$; (4) after the spray dryer was filled with inert gas (nitrogen), adjust the temperature to $65^{\circ} \mathrm{C}$. When the pump velocity reaches a certain value, the precursor mixed solution was pumped to the spray dryer, and RDX/NC/PMMA composite particles were prepared after passing through the cyclone separator.

In order to contrast and analyze the morphology and performance of RDX/NC/PMMA prepared by spray drying method intuitively, the steps of preparing RDX/NC/PMMA composite particles by plain solvent evaporation method are as follows.

Drop a drop of $3 \%$ PVA dispersant into the RDX/NC/PMMA solution, and the solution with ultrasonic oscillation at a frequency of $40 \mathrm{~Hz}$ for 30 minutes, and stirred until the acetone in the solution was completely volatilized at $65^{\circ} \mathrm{C}$. The preparation schematic diagram of the two methods is shown in Figure 1. At the same time, the RDX/NC/PMMA composite particles prepared by the spray drying method were signed as sample 1, and the composite particles prepared by the solvent volatilization method were marked as sample 2 .

\section{Results and Discussion}

3.1. Morphologies of Samples. The RDX raw materials, NC raw materials, and RDX/NC/PMMA composite particles were tested by SEM. The test results were as follows.

As can be seen from Figure 2, NC of raw material presented a coarse rope morphology (as shown in Figure 2(a)), and the crystal surface of raw material RDX demonstrated rough, uneven particle distribution and irregular morphology (as shown in Figure 2(b)). Interestingly (as shown in Figure 2(c)), the RDX/NC/PMMA composites fabricated by spray drying presented spherical particles with uniform distribution and smooth surface. It can be seen intuitively in the insertion diagram of Figure 2(c) that RDX was tightly embedded in the NC/PMMA hybrid system (marked by the red circle in the figure), which manifested that the tension was reduced by the dispersant PVA when the surface of acetone was nucleated during the instant of spray drying. At the same time, the wettability of RDX surface was improved effectively, and the affinity of RDX-NC-PMMA and RDXNC-PMMA was increased up to now. The Hamaker constant also became smaller, and the attraction energy between particles decreased, thus, formed an effective steric hindrance and heightened the dispersion between composite particles. More amusingly, due to the entanglement of NC molecular chains, the above phenomenon did not appear in composite particles prepared by solvent volatilization. It can be seen from Figure 2(d) that the particle size of the composite particles prepared by the solvent volatilization method was smaller than raw material RDX, the morphology was anomalous, and the entirety was in the form of agglomerated particles. Obviously, it can be observed that the prepared particles were piled up (the part marked by the dashed circle in Figure 2(d)). This is because the fluidity of the solution gradually decreases during the solvent volatilization. As the solvent slowly 


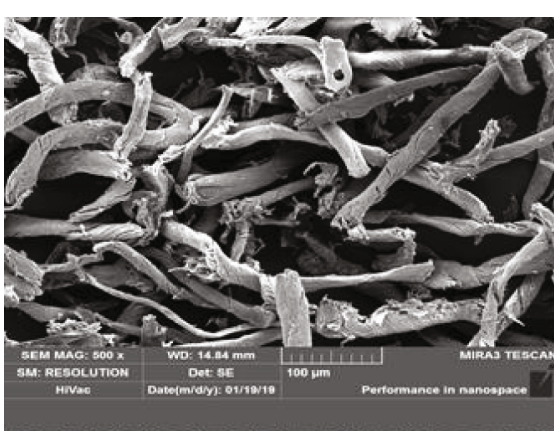

(a)

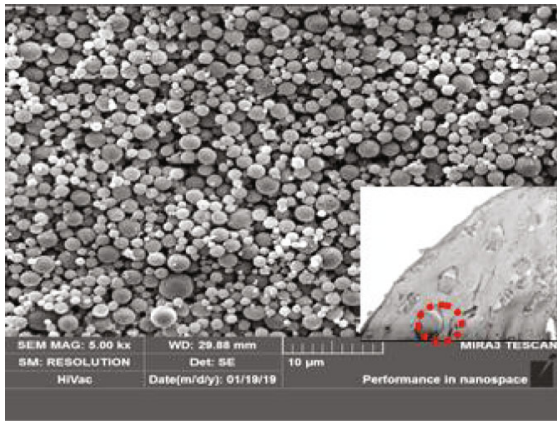

(c)

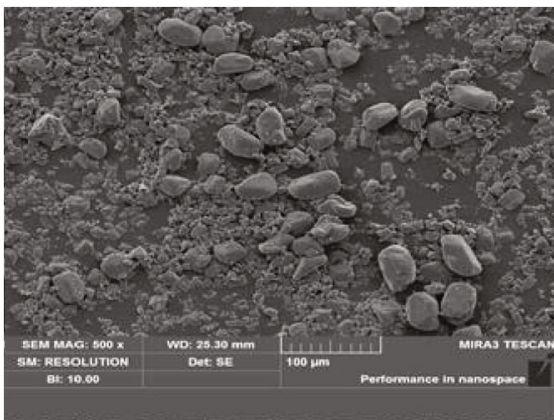

(b)

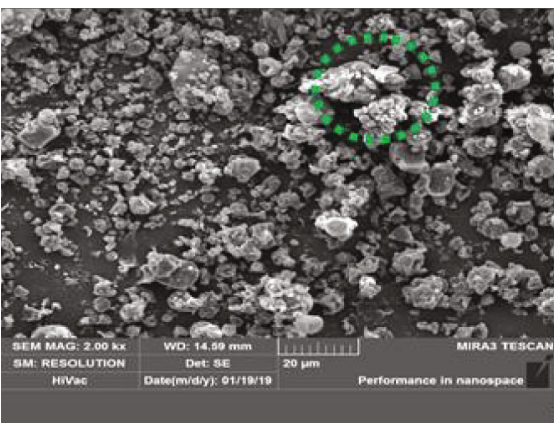

(d)

Figure 2: SEM image of the sample: (a) NC, (b) raw material RDX, (c) RDX/NC/PMMA-1, and (d) RDX/NC/PMMA-2.

evaporates, an increasing number of particles were precipitated, and eventually, a stacking structure was formed.

3.2. Crystal Structure Analysis. In order to study whether the crystal structure of RDX was damaged during the spray drying process, X-ray diffraction analysis was carried out. X-ray diffraction of RDX samples is displayed in Figure 3.

Application of the JADE software is shown in Figure 3. The diffraction peak of RDX/NC/PMMA composite particle could correspond to the main diffraction peak of raw material RDX, and the diffraction peak of NC and PMMA was included by the diffraction peak of RDX/NC/PMMA. This indicates that the crystal structure of RDX was not affected by the addition of NC and PMMA.

Interestingly, it can be observed that the diffraction peak of $2=22.91,25.38$, and 32.32 in the X-ray diffraction diagram has increased in intensity and widens in diffraction width by compared with the PDF card of RDX (JCPDSNO.00-046-1606) (as shown by the enlarged XRD diffraction peak inserted in Figure 3). This is because of the selective preferential orientation of the RDX grain surface growth in the spray drying process after PMMA and NC were added [17]. The process of preparing RDX/NC/PMMA by spray drying method mainly relies on the phase transition (liquid $\rightarrow$ solid) of the binder PMMA and NC, as well as the van der Waals force and hydrogen bond between RDX and PMMA/NC to form a continuous and uniform coating process on the surface of RDX crystal, so the crystal structure will not be destroyed. In addition, RDX underwent a recrystallization growth process during the spray drying process, and the growth of RDX crystals is restricted by the addition of PMMA and NC during the precipitation of RDX crystals. By the Scherrer formula [18]:

$$
D_{\mathrm{hkl}}=\frac{k \lambda}{\beta \cos \theta},
$$

where $D_{\mathrm{hkl}}$ is the grain diameter along the direction perpendicular to the crystal plane; $k$ is the Scherrer constant (usually 0.89 ); $\lambda$ is the incident $\mathrm{X}$-ray wavelength (wavelength is $1.5418 \AA$ ); $\theta$ is the Bragg diffraction angle $\left({ }^{\circ}\right) ; \beta$ is the half-height width of the diffraction peak (rad).

Through calculation, it can be observed that the grain size of RDX in the composite particles increases with the addition of PMMA and NC, which was because the diffraction peak width was affected by the accumulation and growth of NC and PMMA on the RDX surface. The broadening of the diffraction peak width also illustrated the triumphant distribution of NC and PMMA on the surface of RDX.

3.3. Thermal Performance Analysis. The thermal performance of RDX samples was analyzed by DSC curves, the activation energy and preexponential factors of different samples were calculated by linear fitting, the thermal explosion critical temperature of different RDX samples was calculated by formulas, and the DSC curves were shown in Figure 4.

We can observe that there is no thermal event before the RDX endothermic peak for both pure RDX and PMMA/RDX/NC composites in Figure 4, and similar thermal decomposition behaviors have occurred in all three particles. The pure RDX curve shows an endothermic peak 


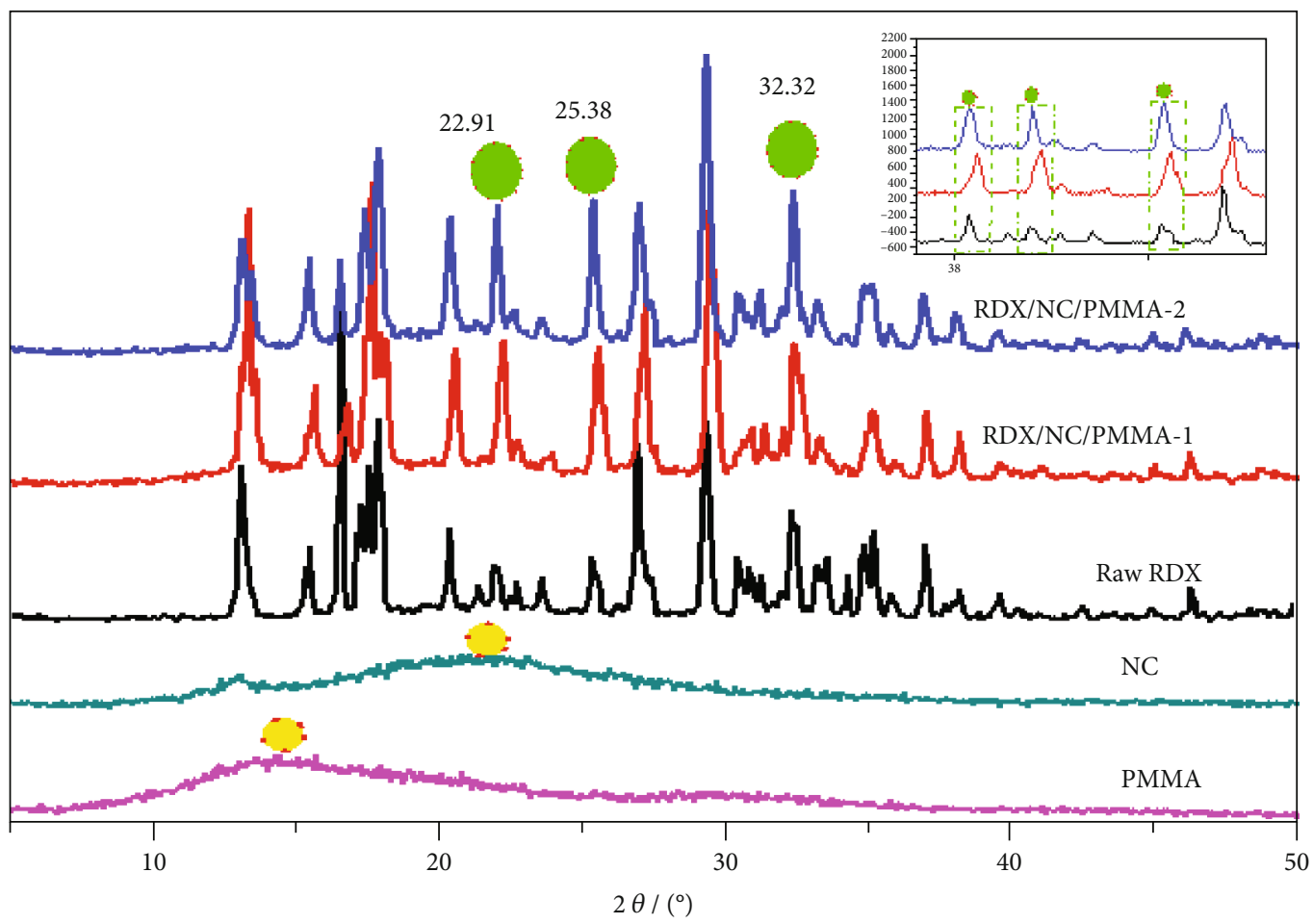

FIGURE 3: XRD pattern of the sample.

followed by an exothermic peak. The sharp endothermic peak corresponds to the melting process at $202.9^{\circ} \mathrm{C}$ to $207.0^{\circ} \mathrm{C}$. We found that the endothermic peaks all have a slight right-shifting trend, which may be due to the increased thermal stability of RDX under the protection of the NC-PMMA shell. The shoulder peak appeared in the DSC curves of RDX, and the size of the shoulder peak was related to the heating feedback and the degree of oxidation-reduction reaction during the thermal decomposition of RDX; interestingly, compared with RDX raw materials, we can evidently observe that the thermal decomposition temperature of PMMA/RDX/NC-1 particles with core-shell structure at a uniform heating rate of $5^{\circ} \mathrm{C} / \mathrm{min}$ has been reduced. This is because the flow temperature of the binder PMMA is about $160^{\circ} \mathrm{C}$, and the flow temperature of another binder NC became very active when it exceeded $40^{\circ} \mathrm{C}$. As the temperature rises, a large number of free radicals in PMMA and NC were decomposed and produced in advance, which induced the premature thermal decomposition of RDX. This situation is consistent with previous reports in the relevant literature [19].

The strange thing is that the thermal decomposition temperature of PMMA/RDX/NC-2 particles decreased smaller than that of PMMA/RDX/NC-1 with the same binder composition. This is obviously because PMMA and $\mathrm{NC}$ are not uniformly distributed on the surface of RDX, and many bare RDX particles were induced by the premature decomposition of PMMA and NC.

According to the decomposition peak temperature of different samples at different heating rates, the apparent activation energies $E_{a 1}, E_{a 2}$, and $E_{a 3}$ of the explosive particles were obtained by Kissinger formula (formula (2)), Ozawa formula (formula (3)), and Starink formula (formula (4)), respectively. The apparent activation energy $\left(E_{a 1}\right)$ was obtained by the Kissinger formula method, and the peak temperature $\mathrm{Tp} 0$ when the heating rate $\beta$ approaches 0 was calculated by formula (5), which was brought into formula (6) to calculate the thermal explosion critical temperature $\mathrm{Tb}[20,21]$. The thermal decomposition kinetic parameters of the samples are shown in Table 1.

$$
\begin{gathered}
\ln \left(\frac{\beta}{T_{p}^{2}}\right)=\ln \left(\frac{\mathrm{AR}}{E_{a}}\right) \frac{E_{a}}{R T_{p}}, \\
\lg \beta=\lg \left(\frac{A E_{a}}{\operatorname{Rg}(a)}\right)-2.315-0.4567 \frac{E_{a}}{R T}, \\
\ln \left(\frac{\beta}{T^{1.8}}\right)=C s-1.0037 \frac{E}{R T}, \\
T_{p i}=T_{p 0}+b \beta_{i}+c \beta_{i}^{2}+d \beta_{i}^{3},
\end{gathered}
$$

where $T_{p}$ is the decomposition peak temperature of the explosive at the heating rate $\beta, \mathrm{K} ; R$ is the gas constant, $8.314 \mathrm{~J} \cdot \mathrm{mol}^{-1} \cdot \mathrm{K}^{-1} ; \beta$ is the heating rate, $\mathrm{K} \cdot \mathrm{min}^{-1} ; A$ is the front factor, $\min ^{-1}$ or $\mathrm{s}^{-1}$; Cs is a constant; $k_{B}$ is the Boltzmann constant with a value of $1.3807 \times 10^{-23} \mathrm{~J} / \mathrm{K}$; and $h$ is a Planck constant with a value of $6.625 \times 10^{-34} \mathrm{~J} \cdot \mathrm{s}$.

It can be seen from Table 1 that compared with the raw material RDX, the activation energy of the RDX/NC/PMMA composite particles prepared by the spray drying method 


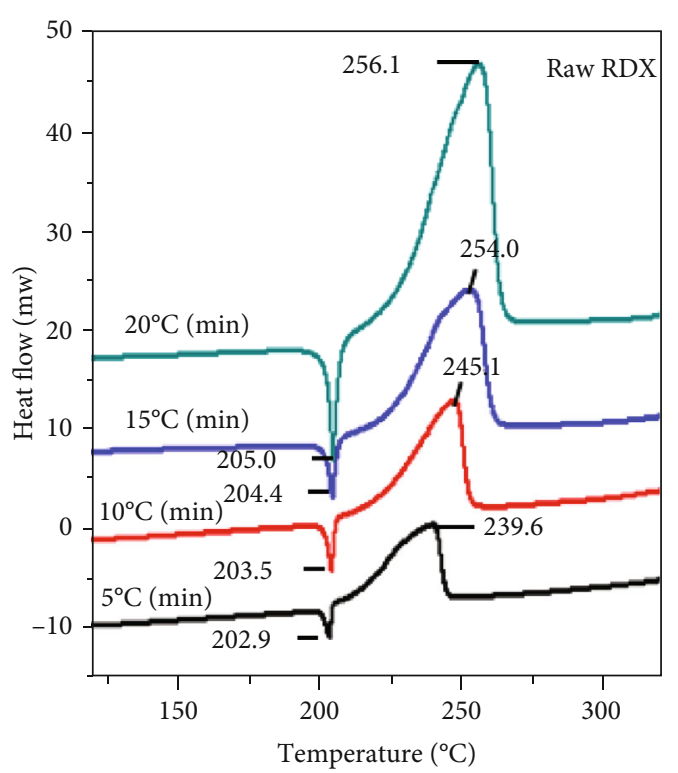

(a)

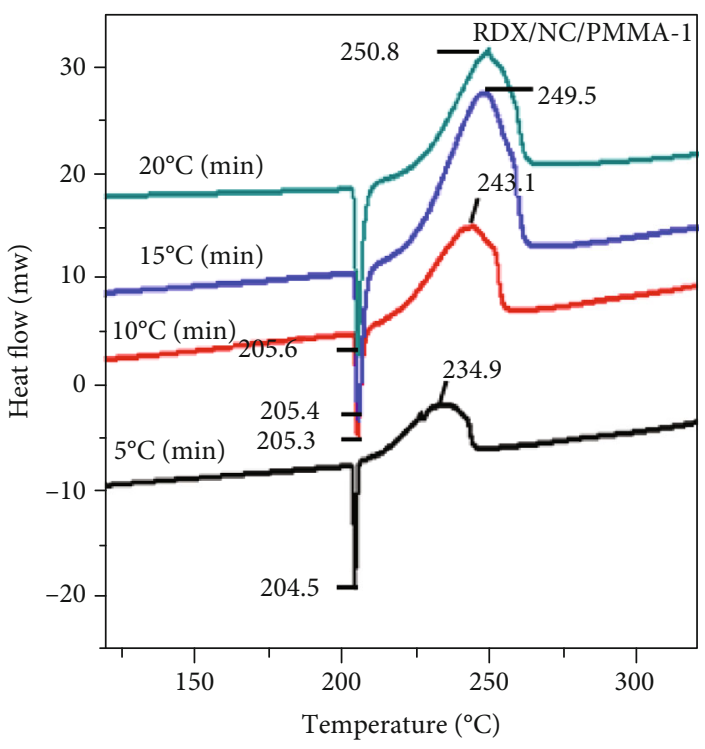

(b)

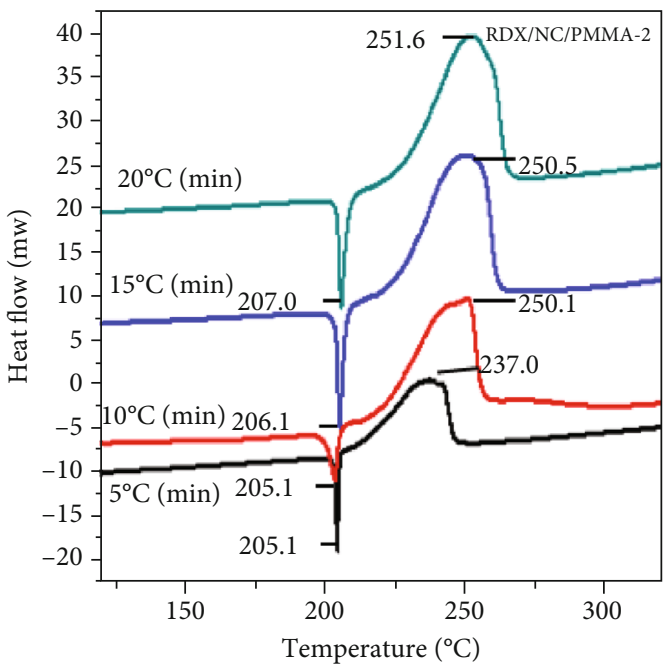

(c)

FIgURE 4: DSC curves of RDX sample.

TABLE 1: Kinetics and thermal stabilities derived from DSC curves.

\begin{tabular}{|c|c|c|c|c|c|c|c|}
\hline \multirow{2}{*}{ Samples } & \multicolumn{6}{|c|}{ Kinetics } & \multirow{2}{*}{$\begin{array}{c}\text { Thermal stabilities } \\
T_{p 0} /{ }^{\circ} \mathrm{C}\end{array}$} \\
\hline & $E_{a 1}$ & $E_{a 2}$ & $E_{a 3}$ & $E_{a}$ & In $A$ & $R$ & \\
\hline Raw RDX & 184.7 & 193.3 & 185.6 & 187.9 & 35.93 & 0.98 & 220.3 \\
\hline RDX/PMMA-1 & 178.2 & 186.8 & 179.0 & 181.3 & 33.99 & 0.99 & 228.2 \\
\hline RDX/PMMA-2 & 161.0 & 169.6 & 161.8 & 164.1 & 30.02 & 0.97 & 214.1 \\
\hline
\end{tabular}

and the solvent volatilization method have been reduced from $187.9 \mathrm{~kJ} / \mathrm{mol}$ to $181.3 \mathrm{~kJ} / \mathrm{mol}$ and $164.1 \mathrm{~kJ} / \mathrm{mol}$. All in all, the addition of PMMA/NC reduced the thermal stability of RDX/NC/PMMA composite particles. It may be because that the PMMA/NC binder was distributed uniformly on the surface of the RDX through the suspension emulsion ball method, which affected the "local chemical" effect of the RDX during the thermal decomposition process, thereby weakening the potential active sites on the RDX surface the reaction center, especially when the thermal decomposition temperature was increased to $200^{\circ} \mathrm{C}$, NC could conduct the greatly violent thermal decomposition in a relatively short time, which ultimately reduced its thermal stability; amusingly, the thermal stability of RDX/NC/PMMA composite particles prepared by spray drying method was better than that of the same component composite particles prepared 


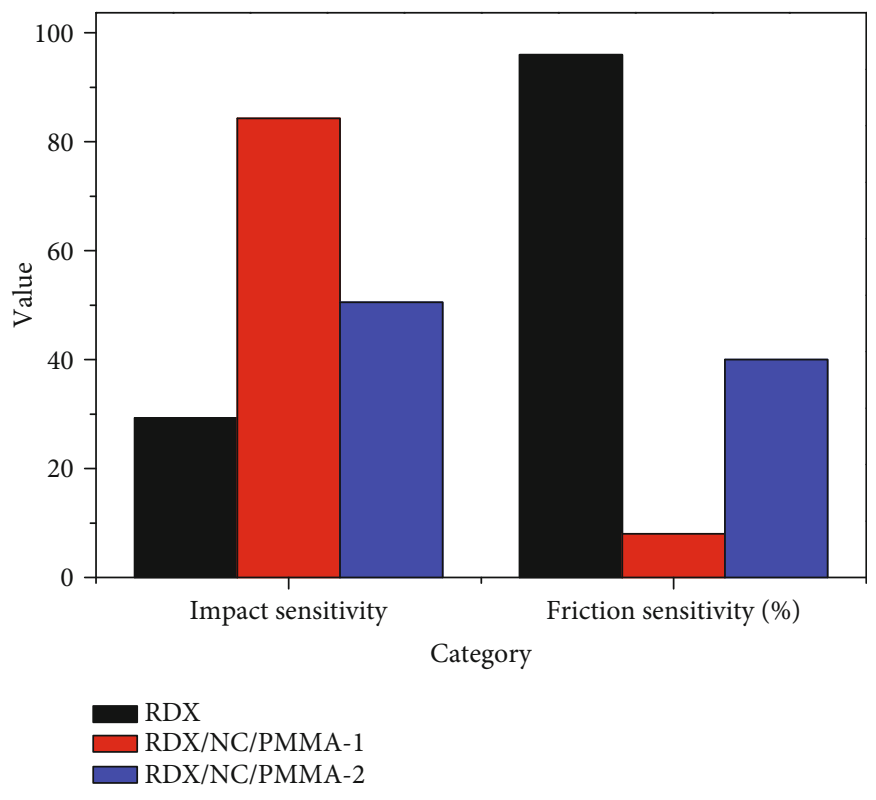

FIgURE 5: Sensitivity histograms for different RDX samples.

by solvent evaporation method. In particular, compared with the raw material RDX, the $T_{p 0}$ of RDX/NC/PMMA-1 increased from $220.3^{\circ} \mathrm{C}$ to $228.2^{\circ} \mathrm{C}$. Obviously, this is affected by the morphology and compactness of RDX/NC/PMMA particles.

\subsection{Impact Sensitivity and Friction Sensitivity Analysis.} Impact sensitivity and friction sensitivity were also pivotal parameters to evaluate the safety performance of energetic materials. In our paper, the impact sensitivity and friction sensitivity of the raw materials RDX, RDX/NC/PMMA-1, and RDX/NC/PMMA-2 samples were tested, and results were presented in Figure 5, which can be observed that the impact sensitivity and friction sensitivity of the composite particles after PMMA/NC coating have been obviously decreased, and the safety performance has been significantly improved. This is because the protruding points on the crystal surface of each elementary explosive were reduced by the addition of PMMA/NC, and the friction coefficient was reduced when the explosive was subjected to local collision or friction, so that the number of hot spots was reduced.

Interestingly, compared with the raw material RDX, the composite particles with the same content of PMMA/NC binder content prepared by spray drying had a tremendous sensitivity reduction effect. The $\mathrm{H}_{50}$ of $\mathrm{RDX} / \mathrm{NC} / \mathrm{PMMA}-1$ and RDX/NC/PMMA-2 composite energetic microspheres increased from $29.32 \mathrm{~cm}$ to $84.3 \mathrm{~cm}$ and $50.5 \mathrm{~cm}$, respectively, and the probability of friction explosion decreased from $96 \%$ to $8 \%$ and $40 \%$, respectively, the safety performance was significantly improved, and the inductive effect is very sensational. Obviously, the safety performance of RDX/NC/PMMA-1 and RDX/NC/PMMA-2 was very varied when used the same component adhesive. This was because the RDX/NC/PMMA composite particles prepared by the spray drying method have a relatively uniform distribution and a smaller particle size distribution, which increased the gap between the particles and the force area of the RDX particles of the same mass, so that the particles the stress concentration phenomenon was reduced, thereby effectively preventing the generation of local hot spots [22, 23].

\section{Conclusion}

Spherical RDX/PMMA/NC composite particles exhibiting obvious spherical effect, uniform particle size distribution, excellent thermal performance, and low sensitivity were prepared via spray drying method, taking PMMA (hydrocarbon binder) with excellent mechanical properties and NC (energetic binder) with higher density as the desensitizing wall material, RDX as the core. The $T_{p 0}$ of RDX/NC/PMMA composite particles was increased from $220.3^{\circ} \mathrm{C}$ to $228.2^{\circ} \mathrm{C}$, significantly enhancing the thermal stability. $\mathrm{H}_{50}$ increased from $29.32 \mathrm{~cm}$ to $84.3 \mathrm{~cm}$, and the probability of friction explosion decreased from $96 \%$ to $8 \%$, which improved the safety performance. The report of RDX/NC/PMMA composite particles prepared by spray drying method has broken the key technical bottleneck that the shell material is easy to separate phases in explosive desensitization coating and improved the technical problems of low coating degree and exposure of explosive particles in desensitization research. Furthermore, the fabrication of RDX/NC/PMMA composite particles expands the application of polymer technology and brings a new way to better solve the contradiction between energy and safety in nitramine explosives simultaneously.

\section{Data Availability}

The data used to support the findings of this study are included within the article. 


\section{Conflicts of Interest}

The authors declare that there is no conflict of interest regarding the publication of this paper.

\section{Acknowledgments}

This work was supported by the Research Fund of State Key Laboratory for Marine Corrosion and Protection, Luoyang Ship Material Research Institute (LSMRI) under the contract (no. 6142901202202), the Natural Science Foundation of Shandong Province for Youth (grant no. ZR2020QE111), and the Research Fund Project of Binzhou University (grant no. BZXYLG1913).

\section{References}

[1] T. J. Tao, J. H. Zhang, E. A. Chi, M. S. Zhao, and Q. Kang, "Study of influence of sensitization process on quality of mixed emulsion explosive," Advanced Materials Research, vol. 10331034, pp. 1305-1308, 2014.

[2] X. Jia and J. Wang, "Preparation and characterization of spherical submicron $\varepsilon$-CL-20 via green mechanical demulsification," Journal of Energetic Materials, vol. 37, no. 4, pp. 475483, 2019.

[3] X. F. Jing, W. Z. Xu, J. Y. Wang, and C. W. An, "Preparation and characterization of spherical $\varepsilon$ CL-20," Journal of North University of China, vol. 352, no. 2, pp. 129-132, 2014.

[4] A. Terracciano, J. Ge, A. Koutsospyros, X. Meng, B. Smolinski, and P. Arienti, "Hexahydro-1,3,5-trinitro-1,3,5-triazine (RDX) reduction by granular zero-valent iron in continuous flow reactor," Environmental Science \& Pollution Research, vol. 25, no. 28, pp. 28489-28499, 2018.

[5] Y. Wang, X. L. Song, S. S. Zhao, and F. S. Li, "Preparation of nano HMX/HNS co/mixed crystal explosive by mechanical ball milling method," Huozhayao Xuebao/Chinese Journal of Explosives and Propellants, vol. 41, no. 3, pp. 261-266, 2018.

[6] Y. Wang, X. Song, D. Song, W. Jiang, H. Liu, and F. Li, “A versatile methodology using sol-gel, supercritical extraction, and etching to fabricate a nitramine explosive: nanometer HNIW," Journal of Energetic Materials, vol. 31, no. 1, pp. 49-59, 2013.

[7] Q. Zhao, X. Ma, W. Yao, and Y. Yao, "A new typhoonmonitoring method using precipitation water vapor," Remote Sensing, vol. 11, no. 23, pp. 2845-2855, 2019.

[8] K. Blahajr, M. Cikrt, L. Kasparova, and M. Jones, "Synergistic enhancement of the biliary excretion of cadmium by the simultaneous administration of two dithiocarbamates," Toxicology Letters, vol. 42, no. 2, pp. 207-213, 1988.

[9] X. Bidault and N. Pineau, "Granularity impact on hotspot formation and local chemistry in shocked nanostructured RDX," The Journal of Chemical Physics, vol. 149, no. 22, pp. 224703224715, 2018.

[10] C. A. Handley, B. D. Lambourn, N. J. Whitworth, H. R. James, and W. J. Belfield, "Understanding the shock and detonation response of high explosives at the continuum and meso scales," Applied Physics Reviews, vol. 5, no. 1, pp. 1130311369, 2018.

[11] J. Shin, A. S. Whittaker, and D. Cormie, "Erratum for "Incident and normally reflected overpressure and impulse for detonations of spherical high explosives in free Air" by Jinwon Shin, Andrew S. Whittaker, and David Cormie," Journal of
Structural Engineering, vol. 146, no. 4, pp. 0822000108220002, 2020.

[12] F. Hernandez, H. Hao, and X. Zhang, "On the effectiveness of ventilation to mitigate the damage of spherical chambers subjected to confined trinitrotoluene detonations," Advances in Structural Engineering, vol. 22, no. 2, pp. 486-501, 2019.

[13] H. R. James and D. B. Hewitt, "Critical energy criterion for the initiation of explosives by spherical projectiles," Propellants Explosives Pyrotechnics, vol. 14, no. 6, pp. 223-233, 1989.

[14] H. Qiu, V. Stepanov, A. R. di Stasio, T. Chou, and W. Y. Lee, "RDX-based nanocomposite microparticles for significantly reduced shock sensitivity," Journal of Hazardous Materials, vol. 185, no. 1, pp. 489-493, 2011.

[15] W. Ji, X. Li, J. Wang, B. Ye, and C. Wang, "Preparation and characterization of the solid spherical HMX/F2602by the suspension spray-drying method," Journal of Energetic Materials, vol. 34, no. 4, pp. 357-367, 2016.

[16] K. Bian, L. Alarid, D. Rosenberg, and H. Fan, "Surfactant assisted self-assembly and synthesis of highly uniform spherical CL-20 microparticles," MRS Advances, vol. 3, no. 41, pp. 2421-2427, 2018.

[17] Y. Guo, X. Quan, N. Lu, H. Zhao, and S. Chen, "High photocatalytic capability of self-assembled nanoporous WO3with preferential orientation of (002) planes," Environmental Science \& Technology, vol. 41, no. 12, pp. 4422-4427, 2007.

[18] X. J. Li, R. Y. Li, X. H. Xie et al., "Research on relation between explosive velocity and ultrafine aluminum oxide size control," Journal-Dalian University of Technology, vol. 47, no. 2, pp. 157-159, 2007.

[19] W. Xu, J. Wang, J. Peng, X. Liang, H. Li, and J. Wang, "Study on the influencing factors of ultrafine spherical RDX during spray drying with low speed," Journal of Nanomaterials, vol. 2019, Article ID 7915129, 10 pages, 2019.

[20] F. G. Wei, M. Enomoto, and K. Tsuzaki, “Applicability of the Kissinger's formula and comparison with the McNabb-Foster model in simulation of thermal desorption spectrum," Computational Materials Science, vol. 51, no. 1, pp. 322-330, 2012.

[21] Y. Li, Z. Yang, J. Zhang et al., "Fabrication and characterization of [email protected] energetic microspheres with reduced sensitivity and superior toughness properties," Composites Science and Technology, vol. 142, pp. 253-263, 2017.

[22] Y. Wang, X. Song, D. Song, L. Liang, C. An, and J. Wang, "Synthesis, thermolysis, and sensitivities of HMX/NC energetic nanocomposites," Journal of Hazardous Materials, vol. 312, pp. 73-83, 2016.

[23] X. L. Jia, L. J. Xu, Y. Y. Hu et al., "Preparation of agglomeration-free composite energetic microspheres taking PMMA-PVA with honeycomb structure as template via the molecular collaborative self-assembly," Journal of Energetic Materials, vol. 39, no. 2, pp. 182-196, 2021. 